Check for updates

Consumers Advancing Patient Safety

Haskell.helen@gmail.com

Cite this as: BMJ 2020;370:m3099

http://dx.doi.org/10.1136/bmi.m3099

Published: 06 August 2020

\section{Cumberlege review exposes stubborn and dangerous flaws in healthcare}

"The healthcare system is disjointed, siloed, unresponsive, and defensive"

Helen Haskell president

On 8 July the Conservative peer Julia Cumberlege published her much anticipated Independent Medicines and Medical Devices Safety Review, looking into the response of England's healthcare system to patients' reports of harm from drugs and medical devices. Commissioned in 2018, the review was conducted through the lens of three medical treatments: hormone pregnancy tests (mainly the drug Primodos), alleged to cause serious birth defects; the anti-epileptic sodium valproate, which can cause birth defects and developmental delays; and pelvic mesh, a surgical material (technically a medical device) implanted in thousands of women to treat organ prolapse and urinary incontinence. Mesh is now the subject of intense global scrutiny after reports of serious long term effects from material that has twisted, moved, disintegrated, or caused severe allergic reactions.

The Cumberlege review was inspired by longstanding patient campaigns alleging harm from these three interventions, and the panel's explicitly declared approach was that patients' and families' voices, experiences, and views should be "at the heart of the review." The published report lives up to that promise. The review panel spoke to over 700 affected individuals, held oral hearings, and received evidence from manufacturers, clinicians, and other stakeholders. They documented pervasive shortcomings in the marketing and oversight of the three treatments and in the response to patients who raised concerns. They considered their findings generalisable across healthcare, as the cover letter states: "We have found that the healthcare system-in which I include the NHS, private providers, the regulators and professional bodies, pharmaceutical and device manufacturers, and policymakers-is disjointed, siloed, unresponsive, and defensive. It does not adequately recognise that patients are its raison d'être."1

Hormone pregnancy tests, sodium valproate, and pelvic mesh have been the subject of regulatory action in the UK and elsewhere. The tests were withdrawn in 1979, 11 years after concerns first emerged. ${ }^{2}$ Sodium valproate, now also used as a psychiatric medication, is the subject of warnings and voluntary restrictions for women of childbearing age, although, as the report documents, it is surprisingly difficult to get this critical information to either patients or doctors. ${ }^{34}$ Mesh for pelvic organ prolapse has been the subject of international campaigns and has recently been withdrawn or advised against in most higher income countries. The mesh bladder sling for urinary incontinence, while "paused" in England, remains available in most other countries. ${ }^{5-8}$ The review found that action in all three cases has been sluggish and inattentive to concerns raised by patients.

Perhaps most striking was the testimony from hundreds of patients reporting lack of informed consent for their initial treatment, followed by years of dismissal by clinicians and regulators who did not want to associate life altering symptoms or injured children with their medical interventions. In a press release Cumberlege said that in years of reviews and inquiries she had never encountered anything like the intensity of suffering of these medically injured families. ${ }^{9}$ The review panel found that healthcare providers' dismissive attitude toward patients was underpinned by a reluctance in all parts of the system to collect evidence on potential harms, by a lack of coordination that would allow clinicians and agencies to interpret and act on that information, and by a culture of denial that failed to acknowledge harm and error, impeding learning and safety.

\section{Familiar pattern}

These patterns are already familiar to all patients who have been harmed by medical care. ${ }^{10}{ }^{11}$ What the Cumberlege team has flagged is the stubborn flaw that lies at the heart of the practice of medicine. It is often called "culture." But this type of embedded attitude seems to go beyond culture, beyond fear of liability, and beyond the profit motive when that exists. It is a patronising and insufficiently curious way of doing business that is often at odds with the realities of helping patients heal and is increasingly out of place in a connected modern world. How to change it is still an unsolved problem. There have been inquiries, reports, and recommendations over the years, but the fundamental issues around power, justice, and compassion are still with us.

The Cumberlege review made nine recommendations (box 1). The first of these, a government apology, has already happened..$^{12}$ The second, a dedicated patient safety commissioner to help patients navigate the bureaucratic tangle and to troubleshoot problems throughout the system, has generated interest both inside and outside England..$^{13}$ It is clearly the panel's centrepiece, characterised as a "golden thread" tying the disjointed system together in the interest of patients. Other recommendations include a Redress Agency to provide remediation to victims of medical harm without forcing them to litigation; schemes and specialist centres to provide care, support, and treatment; reform of the approval and tracking process for drugs and devices; and a public list of doctors' conflicts of interests, to be run by the General Medical Council. 
Box 1: Nine recommendations of the Independent Medicines and Medical Devices Safety Review

- The government should immediately issue a fulsome apology on behalf of the healthcare system to the families affected by Primodos, sodium valproate, and pelvic mesh.

- The appointment of a patient safety commissioner who would be an independent public leader with a statutory responsibility. The commissioner would champion the value of listening to patients and promoting users' perspectives in seeking improvements to patient safety around the use of medicines and medical devices.

- A new independent Redress Agency for those harmed by medicines and medical devices should be created, based on models operating effectively in other countries. The Redress Agency will administer decisions using a non-adversarial process, with determinations based on avoidable harm, looking at systemic failings, rather than blaming individuals.

- Separate schemes should be set up for each intervention-HPTs, valproate, and pelvic mesh-to meet the cost of providing additional care and support to those who have experienced avoidable harm and are eligible to claim.

- Networks of specialist centres should be set up to provide comprehensive treatment, care, and advice for those affected by implanted mesh; and separately for those adversely affected by medications taken during pregnancy.

- The MHRA needs substantial revision, particularly in relation to adverse event reporting and medical device regulation. It needs to ensure that it engages more with patients and their outcomes. It needs to raise awareness of its public protection roles and to ensure that patients have an integral role in its work.

- A central patient-identifiable database should be created by collecting key details of the implantation of all devices at the time of the operation. This can then be linked to specifically created registers to research and audit the outcomes in terms of both the device safety and patient reported outcomes measures.

- Transparency of payments made to clinicians needs to improve. The register of the General Medical Council should be expanded to include a list of financial and non-pecuniary interests for all doctors, as well as doctors' particular clinical interests and their recognised and accredited specialisms. In addition, there should be mandatory reporting for pharmaceutical and medical device industries of payments made to teaching hospitals, research institutions, and individual clinicians.

- The government should immediately set up a task force to implement this review's recommendations. Its first task should be to set out a timeline for their implementation.

The report's final and most important recommendation, that the government urgently set up a task force to implement the first eight recommendations, has not happened, leading to speculation that the report is being buried. ${ }^{14}$ That would be a serious setback. The Cumberlege review has been exceptionally comprehensive, notable for its bird's-eye viewpoint of the family as end user of the system, and for its focus on drugs and devices, which expands the concept of patient safety to include widely misunderstood structural factors that underlie many poor clinical outcomes. This dual vantage point gives the report exceptional power and has the potential to extend its influence far beyond the UK.

But power has no effect without action. Patients and families are waiting to see what happens next.

Commissioning and peer review: Commissioned, not peer reviewed

Competing interests: The BMJ has judged that there are no disqualifying financial ties to commercial companies. The author declares no other interests. The BMJs policy on financial interests is at https://www.bmj.com/sites/default/files/attachments/resources/2016/03/16-current-bmj-educationcoi-form.pdf

1 Cumberlege J. First do no harm: the report of the Independent Medicines and Medical Devices Safety Review. 2020 Jul 8. https://www.immdsreview.org.uk/Report.html.

2 Olszynko-Gryn J, Bjørvik E, Weßel M, Jülich S, Jean C. A historical argument for regulatory failure in the case of Primodos and other hormone pregnancy tests. Reprod Biomed Soc Online 2018;6:34-44. doi: 10.1016/j.rbms.2018.09.003. pmid: 30456319

3 NHS. Medicines: sodium valproate. https://www.nhs.uk/medicines/sodium-valproate.

4 Food and Drug Administration. Drug safety communication 05-06-2013: valproate anti-seizure products contraindicated for migraine prevention in pregnant women due to decreased IQ scores in exposed children. https://www.fda.gov/drugs/drug-safety-and-availability/fda-drug-safetycommunication-valproate-anti-seizure-products-contraindicated-migraine-prevention.

5 European Commission Scientific Committee on Emerging and Newly Identified Health Risks. Opinion on the safety of surgical meshes used in urogynecological surgery. 3 Dec 2015. https://ec.europa.eu/health/scientific_committees/consultations/public_consultations/scenihr_consultation_27_en.

6 Food and Drug Administration. FDA takes action to protect women's health, orders manufacturers of surgical mesh intended for transvaginal repair of pelvic organ prolapse to stop selling all devices. April 2019. https://www.fda.gov/news-events/press-announcements/fda-takes-actionprotect-womens-health-orders-manufacturers-surgical-mesh-intended-transvaginal.

7 Australian Therapeutic Goods Administration. TGA actions after review into urogynaecological surgical mesh implants. https://www.tga.gov.au/alert/tga-actions-after-review-urogynaecologicalsurgical-mesh-implants.

8 New Zealand Medicines and Medical Devices Safety Authority. Medsafe: Regulatory action on surgical mesh products. https://www.medsafe.govt.nz/hot/alerts/UrogynaecologicaSurgicalMeshImplants.asp.

9 Independent Medicines and Medical Devices Safety Review. Press conference speech by Baroness Cumberlege. https://www.immdsreview.org.uk/downloads/IMMDSReview_Baroness_Cumberlege_speech.pdf.

10 Johnson JK, Haskell H, Barach P, eds. Case studies in patient safety: foundations for core competencies. Jones \& Bartlett Learning, 2016.

11 Wailling J, Marshall C, Wilkinson J. Hearing and responding to the stories of survivors of surgical mesh. Ngā kōrero a ngā mōrehu - he urupare. (A report for the Ministry of Health). The Diana Unwin Chair in Restorative Justice. Victoria University of Wellington, 2019.

12 Chalmers V, Pickles K. Matt Hancock apologises to thousands of women "catastrophically" harmed by vaginal mesh, a hormone pregnancy test and an epilepsy treatment after damning report into the three health scandals. Daily Mail. 8 July 2020. https://www.dailymail.co.uk/news/article-8501905/Matt-Hancock-apologises-thousands-women-harmed-vaginal-mesh-primodossodium-valproate.html.

13 Politicians come together to call for patients' commissioner. Sunday Post. 26 Jul 2020. https://www.sundaypost.com/news/scottish-news/politicians-come-together-to-call-for-patientscommissioner/.

14 Why the Cumberlege review is being buried. HSJ Healthcheck Podcast. https://www.hsi.co.uk/hsjhealth-check-podcast/hsj-podcast-why-the-cumberlege-review-is-being-buried/7028015.article. 